\title{
Effects of YuPingFeng granules on acute exacerbations of COPD: a randomized, placebo-controlled study
}

This article was published in the following Dove Press journal: International Journal of COPD

Jinfang Ma,' Jinping Zheng,' Nanshan Zhong,' Chunxue Bai, ${ }^{2}$ Haoyan Wang, ${ }^{3}$ Juan Du, ${ }^{4}$ Fenglei Li, ${ }^{5}$ Yanwei Chen, ${ }^{6}$ Zhe $\mathrm{Shi}^{7}{ }^{7} \mathrm{Xin} \mathrm{Li}^{8}{ }^{8}$ Pingyan Chen ${ }^{9}$

'State Key Laboratory of Respiratory Disease, National Clinical Research Center for Respiratory Disease, Guangzhou Institute of Respiratory Health, the First Affiliated Hospital of Guangzhou Medical University, Guangzhou, People's Republic of China; ${ }^{2}$ Department of Respiratory Medicine, Zhongshan Hospital Affiliated to Fudan University, Shanghai, People's Republic of China; ${ }^{3}$ Department of Respiratory Medicine, Beijing Friendship Hospital Affiliated to Capital Medical University, Beijing, People's Republic of China; ${ }^{4}$ Department of Respiratory Medicine, The Affiliated Hospital of Guizhou Medical University, Guiyang, People's Republic of China; ${ }^{5}$ Department of Respiratory Medicine, The Liwan Hospital of the Third Affiliated Hospital of Guangzhou Medical University, Guangzhou, People's Republic of China; ${ }^{6}$ Department of Respiratory Medicine, Nanshan People's Hospital, Shenzhen, People's Republic of China; ' ${ }^{7}$ epartment of Respiratory Medicine, The First People's Hospital, Huizhou, People's Republic of China; ${ }^{8}$ Department of Respiratory Medicine, China Resource and Wisco General Hospital, Wuhan, People's Republic of China; ' Department of Biostatistics, Southern Medical University, Guangzhou, People's Republic of China

Correspondence: Jinping Zheng: Nanshan Zhong

State Key Laboratory of Respiratory Disease, National Clinical Research Center for

Respiratory Disease, Guangzhou Institute of Respiratory Health, the First Affiliated

Hospital of Guangzhou Medical University,

15I Yan Jiang Rd, Guangzhou 510120 ,

People's Republic of China

Tel +86208306 2869

Fax +862083062729

Email 18928868238@|63.com;

nanshan@vip.163.com
Purpose: Recurrence of acute exacerbations has a major impact on patients with COPD. Therefore, effective prevention and treatment of exacerbation is crucial in the management of COPD, especially for patients with moderate to severe disease. This study evaluated the effectiveness of YuPingFeng granule administration in preventing exacerbation and improving symptom score, as well as its long-term (1 year) safety profile, in patients with COPD.

Patients and methods: This was a randomized, double-blind, parallel, placebo-controlled study of 240 patients from eight centers in China. Participants were eligible if they had mild to severe COPD as defined by Global Initiative for Chronic Obstructive Lung Disease, had a history of at least two COPD exacerbations or one hospitalization within the previous year, and had remained clinically stable for over 4 weeks before the study. They were randomly assigned to receive $5 \mathrm{~g}$ of YuPingFeng or placebo, three times per day, for 1 year. The primary end point was the exacerbation rate over 1 year, and the analysis was by intention to treat. Secondary end points included symptom score, which was assessed by COPD assessment test (CAT) score and safety profiles. This trial was registered in the Chinese Clinical Trial Registry (http://www. chictr.org.cn; registration number: ChiCTR-IPR-15007023).

Results: The YuPingFeng group had a significantly lower exacerbation rate than the placebo group ( 1.15 vs 1.55 ; risk ratio $=0.677$ [95\% CI $0.531-0.863] ; P=0.002)$ and a significantly reduced risk of second exacerbation $(95 \% \mathrm{CI} 0.326-0.772 ; P=0.002)$. After treatment, the mean change in the CAT score in the YuPingFeng group $(-4.41 \pm 7.01)$ differed significantly from that in the placebo group $(-2.49 \pm 5.31 ; P=0.001)$. YuPingFeng was well tolerated.

Conclusion: YuPingFeng granules can be considered as a treatment option for COPD; this treatment prevents acute exacerbations of COPD and has a good safety profile.

Keywords: COPD, exacerbation, treatment, YuPingFeng

\section{Introduction}

COPD is currently the fourth leading cause of death in the world and is estimated to be the disease causing the highest economic burden in China. ${ }^{1}$ Recurrent acute exacerbations of COPD have an even greater impact on patients, resulting in a faster decline of pulmonary function and exponentially increased risk of death. ${ }^{2}$ The Global Initiative for Chronic Obstructive Lung Disease (GOLD) has indicated that the main treatment goal for stable COPD is to relieve the symptoms and reduce the risk of future exacerbations. Therefore, effective prevention and treatment of exacerbations is important in the management of $\mathrm{COPD},{ }^{3}$ particularly in patients with moderate to severe COPD. Many milestone studies of COPD management, such as PEACE, ${ }^{4}$ PANTHEON, ${ }^{5}$ LANTERN,${ }^{6}$ TieCOPD,${ }^{7}$ TORCH,${ }^{8}$ UPLIFT, ${ }^{9}$ WISDOM, ${ }^{10}$ FLAME,${ }^{11}$ 
FULFILL, ${ }^{12}$ and IMPACT, ${ }^{13}$ have been conducted; most of these involved chemical drugs and had exacerbation prevention as a primary or important end point.

Traditional Chinese medicine (TCM) has gained popularity globally and may provide further options for COPD treatment. The TCM "YuPingFeng" has been used in China for over 800 years, and its clinical efficacy and safety are recognized. Unfortunately, few strong evidence-based studies on its use have been published, limiting its wider application. Recently, a number of basic studies have confirmed that YuPingFeng powder (mainly composed of Astragalus, Saposhnikovia divaricata, and Atractylodes) or its extract can significantly improve specific and nonspecific immune function. ${ }^{14-17}$ Clinical studies have demonstrated that YuPingFeng powder reduces the risk of recurrent respiratory infections by regulating the immune system, ${ }^{18}$ inhibiting inflammatory cytokines in vivo, ${ }^{19}$ and by its anti-allergic effects. $^{20}$ Therefore, we hypothesized that YuPingFeng granules may be effective in treating COPD.

The aim of this study was to assess the effectiveness of YuPingFeng granules in patients with COPD in terms of preventing exacerbations and improving symptom score as well as its long-term (1 year) safety profiles.

\section{Patients and methods}

This study was a randomized, stratified, double-blind, parallel, placebo-controlled multicenter clinical study. The study protocol was jointly designed by the principal investigators and the sponsors. To ensure the authenticity of the trial data, all participating investigators were trained before the initiation of the trial (in terms of the protocol, pulmonary function tests, blinding, drug accountability, etc). Beijing Bionovo Medicine Development Co, Ltd, as a third-party contract research organization, was responsible for data collection and management. The Department of Biostatistics of Southern Medical University was responsible for data analysis. This study has been registered on the Chinese Clinical Trial Registry (http://www.chictr.org.cn; registration number: ChiCTR-IPR-15007023).

The study was approved by the local medical ethics committee of the First Affiliated Hospital of Guangzhou Medical University, Beijing Friendship Hospital Affiliated to Capital Medical University, Zhongshan Hospital Affiliated to Fudan University, the Affiliated Hospital of Guizhou Medical University, the Liwan Hospital of the Third Affiliated Hospital of Guangzhou Medical University, Nanshan People's Hospital, the First People's Hospital of Huizhou, and the China Resource and Wisco General Hospital complied with the Declaration of Helsinki and the Good Clinical Practice regulations and was conducted strictly according to the requirements of the trial protocol.

Participants were eligible if they were diagnosed with COPD according to the GOLD 2013 criteria, had a post bronchodilator forced expiratory volume in 1 second $\left(\mathrm{FEV}_{1}\right)$ to forced vital capacity (FVC) ratio $\left(\mathrm{FEV}_{1} / \mathrm{FVC}\right)$ of $<0.7$ and an $\mathrm{FEV}_{1}$ of $30 \%-79 \%$ of the predicted value, were aged 40-80 years, had a history of at least two COPD exacerbations or one hospitalization within the previous 1 year, had remained clinically stable for 4 weeks preceding the study, and had signed the written informed consent form.

Patients were excluded if they had a history of physiciandiagnosed asthma, non-COPD respiratory disorders, pneumonectomy or lung transplantation, required long-term oxygen therapy ( $\geq 12$ hours/day) or pulmonary rehabilitation, showed evidence of alcohol or drug abuse, had known or suspected hypersensitivity to the study medication or some of its ingredients, currently regularly used oral corticosteroids, had severe concomitant heart, liver, or kidney disease, were pregnant or lactating, or had been involved in an investigational drug trial during the previous 3 months.

The primary end point was the exacerbation rate over 1 year. The secondary end points included the following: the time to first and second exacerbations; the time interval and duration between exacerbations, the severity of exacerbation; pulmonary function, and symptom score accessed by COPD assessment test (CAT) score. The safety end points included clinical observations, adverse events, and laboratory test results.

Stratified and block randomization was used to generate a randomization list containing consecutive codes (001-240), with a block size of 4 . A double-blind design was adopted. The drug management personnel dispensed the drugs according to the instructions of the Interactive Web Answering System. Eligible patients were randomly assigned to the YuPingFeng group ( $5 \mathrm{~g}$, three times daily) or the placebo group ( $5 \mathrm{~g}$, three times daily). The study drug (batch number: 150902) and placebo were supplied by Guangdong Medi-World Pharmaceutical Co., Ltd. Foshan, People's Republic of China; they were dissolved in warm water and taken orally. The Chinese medicine granules were manufactured in strict accordance with the standards of the Chinese Pharmacopoeia (2010). The randomized patients were followed up at 12, 24, 36, and 52 weeks. Between visits, study nurses conducted telephonic follow-ups once every 4 weeks. The observation lasted for 52 weeks.

Short-acting or long-acting bronchodilators (including $\beta 2$ agonists, anticholinergics, and theophylline), inhaled 
glucocorticoids, mucolytics, and antitussives used before entering the study were allowed to be continued during the study, but the types of drugs and dosage were to remain unchanged. For patients regularly taking theophylline before the study, the theophylline was replaced by theophylline sustained release tablets 0.2 , every 12 hour, which were used as the basic treatment for patients ${ }^{21}$ who did not use any drugs before entering the study, and no other drugs were allowed.

\section{Statistical analysis}

This study was an exploratory study. Due to the lack of previous reports on similar drugs, no sample size calculation was performed. According to the clinical experience of respiratory specialists and guidance by the academic committee, the patients were randomized based on a 1:1 ratio.

Baseline data and efficacy were analyzed using the modified intention-to-treat (MITT) population, and the safety end points were analyzed using the safety analysis population (SAP). Two-sided tests were used throughout, with the statistical significance level set at 0.05 , and $95 \%$ CIs were used for interval estimation. To compare the primary end point of exacerbation rate, negative binomial regression analysis was used. For between-group comparisons of the time to first exacerbation before and after the treatment, log-rank tests were used for univariate analysis and Cox regression analysis was used for multivariate analysis. For between-group comparisons of the time interval between exacerbations and duration of exacerbation after the treatment, Wilcoxon rank sum test was used for univariate analysis and ANCOVA was used for multivariate analysis. For between-group comparisons of changes in CAT questionnaire scores, paired $t$-tests for two independent samples were used for univariate analysis and ANCOVA was used for multivariate analysis.

\section{Results}

The study was conducted between April 27, 2014, and December 30, 2016. A total of 240 patients, 120 per group, were enrolled from eight study sites. The dropout and exclusion rate was $23.3 \%(28 / 120)$ in the study group and $25.8 \%$ (31/120) in the control group, without significant differences between the groups $\left(\chi^{2}=0.202, P=0.653\right)$. Eventually, 181 patients were included in the per-protocol population. Patient enrollment distribution diagram is shown in Figure 1.

The demographic data, including sex, age, height, weight, body mass index, and smoking history, were not significantly different between the two groups ( $P=0.307$ to $P=0.896$ ). In total, $79.2 \%(95 / 120)$ patients in the study group and

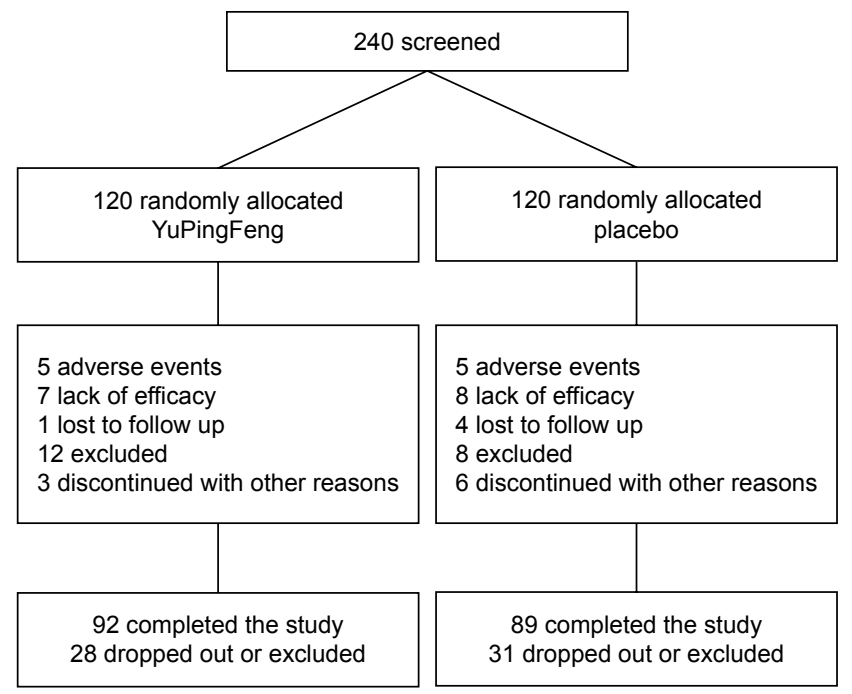

Figure I Patient enrollment distribution diagram.

$80.8 \%(97 / 120)$ in the control group received basic treatment, which was not significantly different $\left(\chi^{2}=0.104\right.$, $P=0.747)$. The two groups were also comparable in terms of the number or severity of exacerbations in the past year, baseline pulmonary function, and CAT score (all $P>0.05$; Table 1).

The mean medication compliance was $90.93 \% \pm 6.96 \%$ in the study group and $90.20 \% \pm 8.34 \%$ in the control group $(t=0.682, P=0.496)$.

After the 52-week treatment, the cumulative number of exacerbations was 129 in the YuPingFeng group and 192 in the placebo group, corresponding to 1.15 exacerbations per patient year with YuPingFeng treatment vs 1.55 with placebo treatment $(P=0.002)$. The number of exacerbations in the YuPingFeng group was significantly lower than that in the placebo group. The risk ratio (RR) of exacerbation was 0.677 (95\% CI $0.531-0.863$ ). Exacerbations of moderate and above occurred at a rate of 1.15 times/person/year in the YuPingFeng group, with a cumulative number of exacerbations of 127, and at a rate of 1.56 times/person/year in the placebo group, with a cumulative number of exacerbations of 167 ( $P=0.038$ ). The between-group exacerbation $\mathrm{RR}$ was 0.765 (95\% CI 0.594-0.986). No interaction was observed with the patient's pulmonary function, smoking status, and time of onset.

Times to first and second exacerbation are shown in Figure $2 \mathrm{~A}$ and $\mathrm{B}$. YuPingFeng had a trend to delay the onset of first exacerbation (median time 31.0 weeks vs 21.0, $P=0.161$ ). Time to second exacerbation was significantly longer in the YuPingFeng group than in the placebo group, with a HR of 0.502 (95\% CI 0.326-0.772), $P=0.002$. 
Table I Comparison of demographic data and baseline conditions between the two groups

\begin{tabular}{|c|c|c|c|c|c|}
\hline \multirow[t]{2}{*}{ Item } & \multirow[t]{2}{*}{ Description } & \multicolumn{2}{|l|}{ Study group } & \multirow[t]{2}{*}{ Statistics } & \multirow[t]{2}{*}{$P$-value } \\
\hline & & YuPingFeng group & Placebo group & & \\
\hline Sex & Male & $109(90.8 \%)$ & $104(86.7 \%)$ & 1.043 & 0.307 \\
\hline Age (years) & & $67.54 \pm 7.37$ & $68.08 \pm 7.37$ & 0.566 & 0.572 \\
\hline Height (cm) & & $165.11 \pm 7.09$ & $164.47 \pm 7.1 \mid$ & 0.704 & 0.482 \\
\hline Weight (kg) & & $60.83 \pm 11.28$ & $61.01 \pm 10.45$ & 0.131 & 0.896 \\
\hline BMI $\left(k g / m^{2}\right)$ & & $22.25 \pm 3.53$ & $22.54 \pm 3.49$ & 0.623 & 0.534 \\
\hline CAT score & & $15.18 \pm 7.42$ & $15.13 \pm 6.88$ & 0.063 & 0.950 \\
\hline Smoking history & Smoking & 57 (47.5\%) & $53(44.2 \%)$ & 0.269 & 0.604 \\
\hline Pulmonary function & $\mathrm{FEV}_{1}(\mathrm{~L})$ & $1.17 \pm 0.35$ & $1.13 \pm 0.38$ & 1.201 & 0.230 \\
\hline \multirow[t]{2}{*}{ mean $\pm S D$} & FVC (L) & $2.36 \pm 0.67$ & $2.36 \pm 0.74$ & 0.352 & 0.725 \\
\hline & $\mathrm{FEV}_{1} / \mathrm{FVC}(\%)$ & $50.85 \pm$ II. 23 & $49.25 \pm I I .05$ & 0.917 & 0.359 \\
\hline Number of exacerbations & All exacerbations & $1.88 \pm 0.61$ & $1.94 \pm 0.75$ & -0.338 & 0.736 \\
\hline \multirow[t]{2}{*}{ in the past year } & Grade II and above exacerbations & $\mathrm{I} .5 \mathrm{I} \pm 0.88$ & $1.57 \pm 0.92$ & -0.460 & 0.645 \\
\hline & Grade III exacerbations & $0.53 \pm 0.67$ & $0.64 \pm 0.76$ & -1.060 & 0.289 \\
\hline \multirow[t]{7}{*}{ Basic treatment } & $\beta_{2}$ receptor agonist & $36(30.0 \%)$ & $4 \mathrm{I}(34.2 \%)$ & 0.478 & 0.489 \\
\hline & Anticholinergic agent & 50 (4I.7\%) & 50 (4I.7\%) & 0.000 & 1.000 \\
\hline & Theophylline & 39 (32.5\%) & $43(35.8 \%)$ & 0.296 & 0.586 \\
\hline & Inhaled glucocorticoids & $48(40.0 \%)$ & $50(41.7 \%)$ & 0.069 & 0.793 \\
\hline & Mucolytics & $34(28.3 \%)$ & $29(24.2 \%)$ & 0.538 & 0.463 \\
\hline & Antitussives & $5(4.2 \%)$ & $2(1.7 \%)$ & 1.324 & 0.250 \\
\hline & Others & $26(21.7 \%)$ & $29(24.2 \%)$ & 0.212 & 0.645 \\
\hline
\end{tabular}

Abbreviations: BMI, body mass index; CAT, COPD assessment test.

The time interval between exacerbations was also compared between the groups. The median time interval was 94 days (25th percentile [P25]-75th percentile [P75]: 42.0-168.0 days) in the YuPingFeng group and 71.5 days (P25-P75 34.0-131.5 days) in the placebo group, with a between-group difference of 21.6 days (95\% CI 2.5-40.7 days) after adjustment for baseline and site, which was statistically significant $(P=0.027)$.

\section{Symptom score}

After 52 weeks of treatment, the average change in the CAT score was $-4.41 \pm 7.01$ in the YuPingFeng group and $-2.49 \pm 5.31$ in the placebo group. After adjusting for baseline and site, the between-group difference value was -2.101 (95\% CI -3.376 to -0.826$)$, with a statistically significant difference between the groups ( $P=0.001$; Table 2).

\section{Pulmonary function}

No significant differences were found between groups in terms of the pulmonary function as measured by $\mathrm{FEV}_{1}, \mathrm{FVC}$, and $\mathrm{FEV}_{1} / \mathrm{FVC}$ before and after the treatment (Table 3).

\section{Safety}

There were six (5\%) drug-related adverse events that occurred in six patients in the YuPingFeng group and
$22(18.3 \%)$ drug-related adverse events that occurred in 13 patients in the placebo group, with no significant difference between the groups ( $P=0.647$; Table 4$)$. The incidence of serious adverse events was $39.2 \%$ (47/120) in the study group and $34.2 \%(79 / 120)$ in the control group, which was not statistically significantly different $(P=0.422)$.

\section{Discussion}

The results of this study demonstrated that YuPingFeng granules significantly reduced exacerbation compared with placebo. The exacerbation rate over 1 year (1.15 vs 1.55$)$ in both groups was similar to that reported in the PEACE study $(1.01 \text { vs } 1.35)^{4}$ and the PANTHEON study (1.16 vs 1.49$) .{ }^{5}$ The incidence of exacerbation was reduced by $32.3 \%$ by YuPingFeng treatment. YuPingFeng granules had a similar effect in preventing exacerbation as the bronchodilators that are widely used for stable COPD, including long-acting $\beta$ adrenoceptor agonists, long-acting muscarinic receptor antagonists, and inhaled corticosteroids. ${ }^{22}$

Our study showed that YuPingFeng can prevent the risk of second exacerbations. Therefore, attention should be given not only to the overall exacerbation rate but also to the exacerbation recurrence rate, which may be more instructive for clinical treatment. As shown in the ECLIPSE study, ${ }^{23}$ the frequency of exacerbations strongly predicts the possibility of future acute exacerbations: the risk of exacerbation increased 5.72 times in individuals in whom 
A

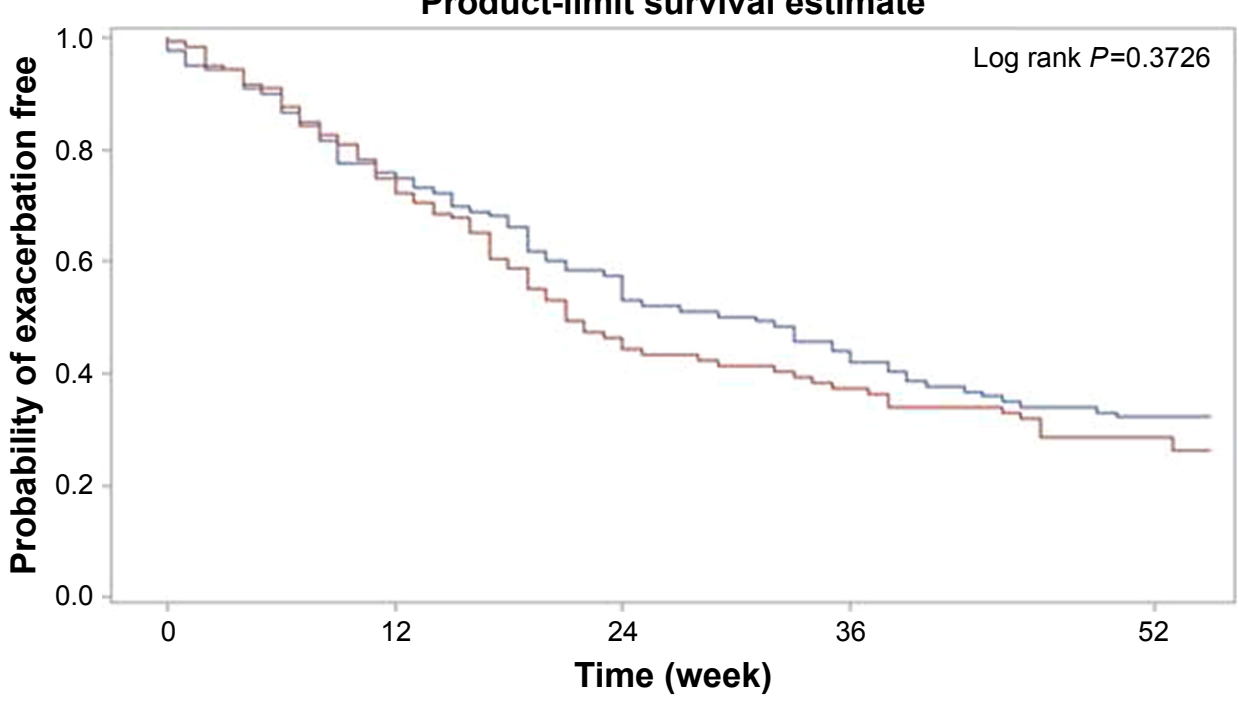

\begin{tabular}{|c|c|c|c|c|}
\hline & & & & \\
\hline & \multicolumn{3}{|c|}{ Group $=$ YPF $=$ Placebo } \\
\hline Drug & 120 & 89 & 64 & 49 \\
\hline Placebo & 120 & 86 & 48 & 34 \\
\hline
\end{tabular}

B

Product-limit survival estimate

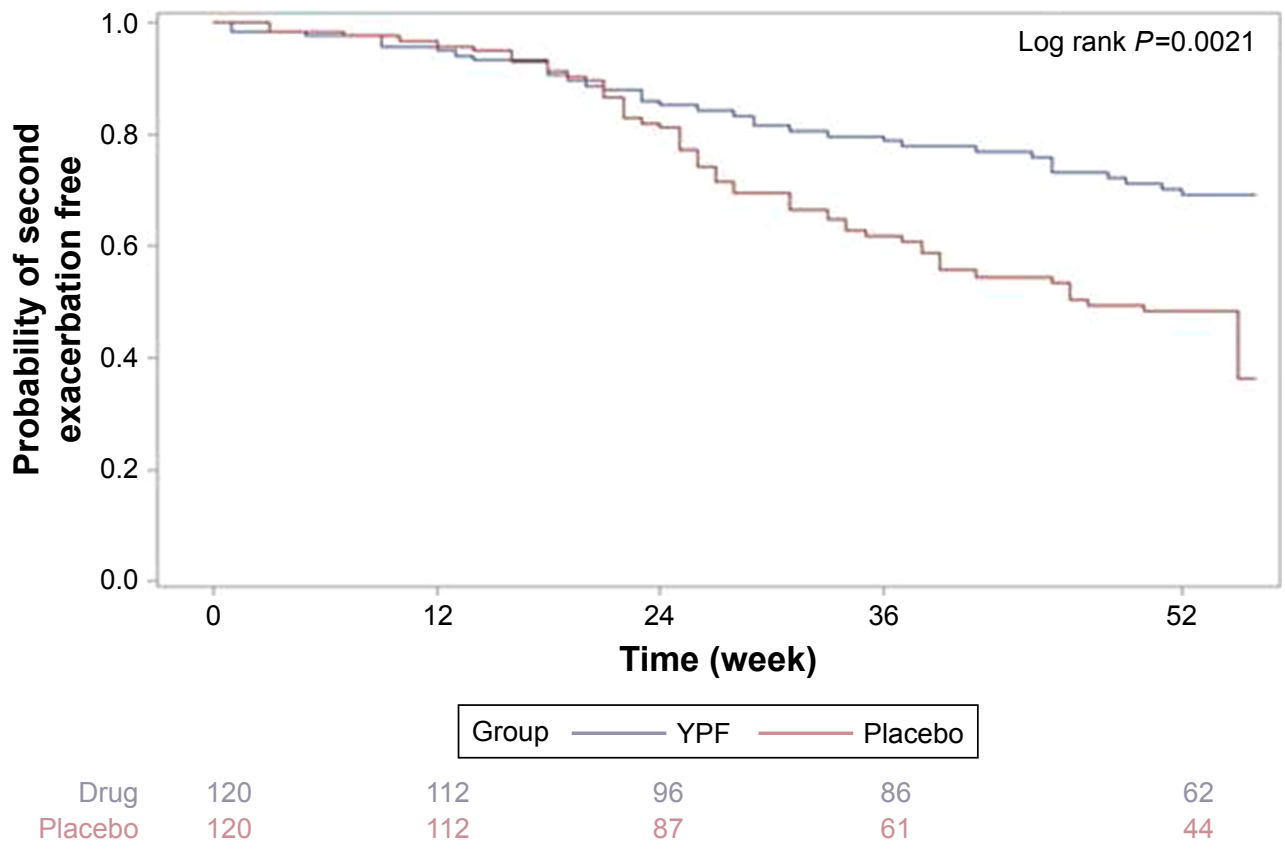

Figure 2 Comparison of time to exacerbation event in patient receiving YuPingFeng or placebo.

Notes: (A) Comparison of time to first exacerbation. (B) Comparison of time to second exacerbation.

Abbreviation: YPF, YuPingFeng.

Table 2 Intragroup and intergroup comparison of CAT scores in the two groups

\begin{tabular}{llll}
\hline CAT score & $\begin{array}{l}\text { YuPingFeng } \\
\text { group }\end{array}$ & $\begin{array}{l}\text { Placebo } \\
\text { group }\end{array}$ & P-value \\
\hline Baseline & $15.13 \pm 7.50$ & $15.13 \pm 6.88$ & \\
52 weeks & $10.30 \pm 6.31$ & $12.95 \pm 5.99$ & $<0.00 \mathrm{I}$ \\
Difference value & $-4.41 \pm 7.0 \mathrm{I}$ & $-2.49 \pm 5.3 \mathrm{I}$ & $0.00 \mathrm{I}$ \\
\hline
\end{tabular}

Note: Data presented as mean \pm SD.

Abbreviation: CAT, COPD assessment test. more than two exacerbations occurred in the previous year. YuPingFeng prolonged the time to onset of first exacerbation (31.0 vs 21.0 weeks) and the exacerbation interval (94.0 vs 71.5 days), which agreed with the reduced exacerbation rate in the YuPingFeng group.

YuPingFeng granules are marketed as a TCM preparation consisting of $S$. divaricata, Astragalus, and Atractylodes. High-performance liquid chromatography-mass spectrometry 
Table 3 Statistical description of pulmonary function indices of the two groups

\begin{tabular}{lllllll}
\hline Index & Visit & Group & N & Mean & SD & P-value \\
\hline FEV & Baseline & YuPingFeng & 120 & 1.17 & 0.35 & - \\
& & Placebo & 120 & 1.13 & 0.38 & - \\
& \multirow{2}{*}{52 weeks } & YuPingFeng & 97 & 1.21 & 0.49 & 0.417 \\
& & Placebo & 87 & 1.15 & 0.40 & 0.779 \\
FVC & \multirow{2}{*}{ Baseline } & YuPingFeng & 120 & 2.36 & 0.67 & - \\
& & Placebo & 120 & 2.36 & 0.74 & - \\
& \multirow{2}{*}{52 weeks } & YuPingFeng & 97 & 2.36 & 0.71 & 0.677 \\
& & Placebo & 87 & 2.34 & 0.65 & 0.412 \\
FEV /FVC\% & \multirow{2}{*}{ Baseline } & YuPingFeng & 120 & 50.85 & 11.23 & - \\
& & Placebo & 120 & 49.25 & 11.05 & - \\
& \multirow{2}{*}{52 weeks } & YuPingFeng & 97 & 51.68 & 13.61 & 0.507 \\
& & Placebo & 87 & 49.59 & 11.94 & 0.920 \\
\hline
\end{tabular}

Abbreviations: $\mathrm{FEV}_{1}$, forced expiratory volume in I second; FVC, forced vital capacity.

was performed by $\mathrm{Li}$ et $\mathrm{al}^{24}$ to analyze the chemical components of 11 batches of YuPingFeng, and the fingerprint chromatogram of the batches was highly similar, with a total of 14 main fingerprint peaks determined at $254 \mathrm{~nm}$; there were no significant changes in the relative SDs of relative retention time and relative peak area.

Pharmacological studies have confirmed that the longterm use of Astragalus can improve the body's immune function. ${ }^{25}$ Atractylodes can significantly ameliorate metabolic status, shows anti-thermal stress activities, and improves cellular immune function. ${ }^{26}$ Atractylodes also has anti-inflammatory and anti-spasmodic effects. ${ }^{27}$ The results of a dexamethasone-induced immunosuppression rat model study $^{24}$ showed that YuPingFeng can regulate nonspecific

Table 4 Comparison of drug-related adverse reactions between the two groups

\begin{tabular}{lll}
\hline & $\begin{array}{l}\text { YuPingFeng group } \\
\text { (120 patients) }\end{array}$ & $\begin{array}{l}\text { Placebo group } \\
\text { ( } 20 \text { patients) }\end{array}$ \\
\hline Gingivitis & 2 & 2 \\
Toothache & 1 & \\
Dizziness & 2 & 2 \\
Insomnia & & 3 \\
AECOPD & $\mathrm{I}$ & 3 \\
Pharyngitis & & $\mathrm{I}$ \\
Dry throat & & $\mathrm{I}$ \\
Cough & & $\mathrm{I}$ \\
Excessive phlegm & & $\mathrm{I}$ \\
Impaired vision & & $\mathrm{I}$ \\
Back pain & & 3 \\
Diarrhea & & 2 \\
Constipation & & 2 \\
Gastritis & &
\end{tabular}

Abbreviation: AECOPD, acute exacerbations of COPD. immunity in mice by improving the growth of $\mathrm{T}$ and $\mathrm{B}$ cells and inhibiting lymphocyte apoptosis. It also regulates cellular immunity by improving the CD4/CD8 cell ratio, NK cell activity, and macrophage phagocytosis. A meta-analysis on YuPingFeng treatment of recurrent respiratory tract infections in children also provided some insights. ${ }^{28} \mathrm{~A}$ total of 1,236 subjects in 12 randomized control trials (RCTs) were screened, with a 4-week to 3-month treatment period and a 6-12-month observation period. Compared with traditional therapy alone, combined treatment with YuPingFeng significantly increased serum levels of $\operatorname{IgA}, \operatorname{IgG}, \operatorname{IgM}$, and $\mathrm{CD}^{+}$cells, and three of the studies demonstrated that treatment with YuPingFeng reduced the frequency of recurrent respiratory tract infections in children. Therefore, we speculate that the reduction in the exacerbation frequency of patients with COPD by YuPingFeng may be related to its ability to improve immunity and reduce recurrent respiratory tract infections. The current study further showed that the use of YuPingFeng granules as an adjunct to regular treatment can alleviate the clinical symptoms and improve the symptom score of patients with COPD. This may be related to the antifatigue and hypoxia-resistant functions of Astragalus. ${ }^{29}$ No significant changes were found in pulmonary function as measured by $\mathrm{FEV}_{1}, \mathrm{FVC}, \mathrm{FEV}_{1} / \mathrm{FVC}$, and other indicators in the two groups before and after the treatment. We concluded that YuPingFeng granules have little effect on bronchodilation.

In terms of safety, the results of this study showed that the incidence of drug-related adverse events was similar in the treatment and placebo groups, and the events recorded were mainly gingivitis and dizziness. Consistent with our findings, there have been relatively few clinical reports of adverse events of YuPingFeng granules to date. The adverse events mainly involved gastrointestinal disorder, such as diarrhea and abdominal pain, and nervous system issues, involving dizziness, headache, gingival pain, and swelling, and dry throat. The serious adverse events reported were mainly hospitalizations due to exacerbation. One patient died (in a car accident), and two patients died of AECOPD, but these were not considered to be related to the study drug.

Previous clinical studies on the treatment of COPD with TCM have also reported a reduction in acute exacerbations. ${ }^{30,31}$ However, these studies had certain design shortcomings, such as relatively short treatment cycles, ${ }^{30,31}$ a lack of randomized or double-blind design, ${ }^{30}$ or a lack of reporting on concomitant medications. ${ }^{31}$ To the best of our knowledge, no previous study has reported a multicenter, randomized, double-blind, controlled clinical trial of the effect of YuPingFeng granules 
on the treatment of stable COPD. The current multicenter study used a sufficiently long period ( 52 weeks) to eliminate the effect of seasonal changes on exacerbation. The entire study process was strictly according to the protocol and the requirements of good clinical practice. However, this study also had some limitations, such as not assessing changes in relevant inflammatory indicators during the treatment, and the molecular targets, mechanism of action, and the active ingredients of YuPingFeng were not elucidated. Given its proven clinical efficacy, further research on its mechanism of action and active ingredients is needed in future. Clinical trials with larger sample sizes are also warranted to confirm the results of our study.

\section{Conclusion}

This study showed that the use of YuPingFeng granules for 52 weeks, in conjunction with conventional treatment, significantly reduced the risk of moderate or severe acute exacerbations and improved the symptom score in patients with moderate to severe COPD, indicating that it has certain preventive effects against exacerbation. The study drug has a good safety profile, is conveniently dissolved and taken orally, has a pleasant taste, engendering good medication compliance, and has a relatively low cost, and therefore should be considered for wider clinical application for the treatment of patients with stable COPD. Our study results suggest that YuPingFeng granules may play an important role in TCM treatment of COPD in future.

\section{Acknowledgments}

We would like to thank the patients for participating in this study, the study investigators and their staff, and the study team. This study was funded by Guangdong Medi-World Pharmaceutical Co., Ltd. We would also like to thank Editage (www.editage.cn) for English language editing.

\section{Author contributions}

JM, JZ, and NZ contributed to study design, quality control, and drafting and revising of the manuscript. JM, CB, HW, JD, FL, YC, ZS, and XL contributed to acquisition and the accuracy of the data. PC contributed to statistical analysis. All authors contributed toward data analysis, drafting and revising the paper and agree to be accountable for all aspects of the work.

\section{Disclosure}

The authors report no conflicts of interest in this work.

\section{References}

1. Vogelmeier CF, Criner GJ, Martinez FJ, et al. Global Strategy for the Diagnosis, Management, and Prevention of Chronic Obstructive Lung Disease 2017 Report. GOLD Executive Summary. Am J Respir Crit Care Med. 2017;195(5):557-582.

2. Wedzicha JA, Singh R, Mackay AJ. Acute COPD exacerbations. Clin Chest Med. 2014;35(1):157-163.

3. Sethi S, Mahler DA, Marcus P, Owen CA, Yawn B, Rennard S. Inflammation in COPD: implications for management. Am J Med. 2012; 125(12):1162-1170.

4. Zheng JP, Kang J, Huang SG, et al. Effect of carbocisteine on acute exacerbation of chronic obstructive pulmonary disease (PEACE Study): a randomised placebo-controlled study. Lancet. 2008;371(9629): 2013-2018.

5. Zheng JP, Wen FQ, Bai CX, et al. Twice daily N-acetylcysteine $600 \mathrm{mg}$ for exacerbations of chronic obstructive pulmonary disease (PANTHEON): a randomised, double-blind placebo-controlled trial. Lancet Respir Med. 2014;2(3):187-194.

6. Zhong N, Wang C, Zhou X, et al. LANTERN: a randomized study of QVA149 versus salmeterol/fluticasone combination in patients with COPD. Int J Chron Obstruct Pulmon Dis. 2015;10:1015-1026.

7. Zhou Y, Zhong NS, Li X, et al. Tiotropium in Early-Stage Chronic Obstructive Pulmonary Disease. N Engl J Med. 2017;377(10):923-935.

8. Calverley PM, Anderson JA, Celli B, et al. Salmeterol and fluticasone propionate and survival in chronic obstructive pulmonary disease. N Engl J Med. 2007;356(8):775-789.

9. Tashkin DP, Celli B, Senn S, et al. A 4-year trial of tiotropium in chronic obstructive pulmonary disease. $N$ Engl J Med. 2008;359(15): $1543-1554$.

10. Magnussen H, Disse B, Rodriguez-Roisin R, et al. Withdrawal of inhaled glucocorticoids and exacerbations of COPD. $N$ Engl $J$ Med. 2014;371(14):1285-1294

11. Wedzicha JA, Zhong N, Ichinose M, et al. Indacaterol/glycopyrronium versus salmeterol/fluticasone in Asian patients with COPD at a high risk of exacerbations: results from the FLAME study. Int $J$ Chron Obstruct Pulmon Dis. 2017;12:339-349.

12. Lipson DA, Barnacle H, Birk R, et al. FULFIL trial: once-daily triple therapy for patients with chronic obstructive pulmonary disease. Am J Respir Crit Care Med. 2017;196(4):438-446.

13. Lipson DA, Barnhart F, Brealey N, et al. Once-daily single-inhaler triple versus dual therapy in patients with COPD. $N$ Engl $J$ Med. 2018;378(18):1671-1680

14. Jin H, Wang L, Li B, et al. Astragaloside IV ameliorates airway inflammation in an established Murine model of asthma by inhibiting the mTORC1 signaling pathway. Evid Based Complement Alternat Med. 2017;2017:4037086:1-10

15. Sun H, Ni X, Zeng D, Hao S, Dong Z, et al. Bidirectional immunomodulating activity of fermented polysaccharides from Yupingfeng Res Vet Sci. 2017;110:22-28.

16. Wang Z, Cai X, Pang Z, et al. Yupingfeng Pulvis regulates the balance of $\mathrm{T}$ cell subsets in asthma mice. Evid Based Complement Alternat Med. 2016;2016:6916353.

17. Yang ZS, Yan JY, Han NP, et al. Anti-inflammatory effect of Yu-PingFeng-San via TGF- $\beta 1$ signaling suppression in rat model of COPD. Iran J Basic Med Sci. 2016;19(9):993-1002.

18. Ma XQ. Clinical effect of Yupingfeng particle as an adjuvant treatment for recurrent respiratory tract infections in children. $J$ Practical Med. 2012;28:1000-1001.

19. Chan PH, To CY, Chan EY, et al. A randomized placebo-controlled trial of traditional Chinese medicine as an add-on therapy to oral montelukast in the treatment of mild persistent asthma in children. Complement Ther Med. 2016;29:219-228.

20. Luo Q, Zhang CS, Yang L, et al. Potential effectiveness of Chinese herbal medicine Yu ping feng san for adult allergic rhinitis: a systematic review and meta-analysis of randomized controlled trials. BMC Complement Altern Med. 2017;17(1):485. 
21. Zhou Y, Wang X, Zeng X, et al. Positive benefits of theophylline in a randomized, double-blind, parallel-group, placebo-controlled study of low-dose, slow-release theophylline in the treatment of COPD for 1 year. Respirology. 2006;11(5):603-610.

22. Wedzicha JA, Calverley PM, Seemungal TA, et al. The prevention of chronic obstructive pulmonary disease exacerbations by salmeterol/fluticasone propionate or tiotropium bromide. Am J Respir Crit Care Med. 2008;177(1):19-26.

23. Hurst JR, Vestbo J, Anzueto A, et al. Susceptibility to exacerbation in chronic obstructive pulmonary disease. N Engl J Med. 2010;363(12): 1128-1138.

24. Li Y, Zheng B, Tian H, et al. Yupingfeng Powder relieves the immune suppression induced by dexamethasone in mice. J Ethnopharmacol. 2017;200:117-123.

25. du X, Zhao B, Li J, et al. Astragalus polysaccharides enhance immune responses of HBV DNA vaccination via promoting the dendritic cell maturation and suppressing Treg frequency in mice. Int Immunopharmacol. 2012;14(4):463-470.

26. Xu D, Li W, Huang Y, He J, Tian Y. The effect of selenium and polysaccharide of Atractylodes macrocephala Koidz. (PAMK) on immune response in chicken spleen under heat stress. Biol Trace Elem Res. 2014;160(2):232-237.
27. Kong X, Liu C, Zhang C, et al. The suppressive effects of Saposhnikovia divaricata (Fangfeng) chromone extract on rheumatoid arthritis via inhibition of nuclear factor- $\mathrm{KB}$ and mitogen activated proteinkinases activation on collagen-induced arthritis model. J Ethnopharmacol. 2013;148(3):842-850.

28. Song T, Hou X, Yu X, et al. Adjuvant treatment with Yupingfeng formula for recurrent respiratory tract infections in children: a metaanalysis of randomized controlled trials. Phytotherapy Research. 2016;30(7):1095-1103.

29. Yeh TS, Chuang HL, Huang WC, Chen YM, Huang CC, Hsu MC. Astragalus membranaceus improves exercise performance and ameliorates exercise-induced fatigue in trained mice. Molecules. 2014; 19(3):2793-2807.

30. Li SY, Li JS, Wang MH, et al. Effects of comprehensive therapy based on traditional Chinese medicine patterns in stable chronic obstructive pulmonary disease: a four-center, open-label, randomized, controlled study. BMC Complement Altern Med. 2012;12:1-11.

31. Wang G, Liu B, Cao Y, et al. Effects of two Chinese herbal formulae for the treatment of moderate to severe stable chronic obstructive pulmonary disease: a multicenter, double-blind, randomized controlled trial. PLoS One. 2014;9(8):1-12.
International Journal of COPD

\section{Publish your work in this journal}

The International Journal of COPD is an international, peer-reviewed journal of therapeutics and pharmacology focusing on concise rapid reporting of clinical studies and reviews in COPD. Special focus is given to the pathophysiological processes underlying the disease, intervention programs, patient focused education, and self management protocols.

\section{Dovepress}

This journal is indexed on PubMed Central, MedLine and CAS. The manuscript management system is completely online and includes a very quick and fair peer-review system, which is all easy to use. Visit http://www.dovepress.com/testimonials.php to read real quotes from published authors. 